\title{
COMPROBACIÓN DE LA LONGITUD DE PANDEO, DADA POR LA MV-103 DE 1972, O POR LA E.A. 95, CON LA SOLUCIÓN DIFERENCIAL
}

\author{
(CHECKING BULGING LENGTH ESTABLISHED BY STANDARDS MV-103 AND E. A. 95, WITH \\ THE DIFFERENTIAL SOLUTION)
}

Pedro Amondaráin Elícegui, Dr. Ingeniero Industrial

Susana Villanueva Besga, Ingeniero Técnico Industrial

Fecha de recepción: 29-XII-97

400-32

ESPAÑA

RESUMEN

SUMMARY

Se estudia y comprueba en este artículo la longitud de pandeo, dada a través de las Normas MV-103 y E. A. 95 para el cálculo y diseño de estructuras metálicas. El estudio pone de manifiesto los errores en dichas Normas, en concreto en lo que respecta a los valores del coeficiente $\beta$.

The bulging length by Standards MV-103 and E. A. 95 is studied and reckoned aiming the calculation and design of metallic structures. The study evidences the errors contained in Standards specially regarding coefficient $\beta$ values.

\section{INTRODUCCIÓN}

Un tema importante en el cálculo de las estructuras metálicas es el pandeo.

Las piezas comprimidas, junto a las que trabajan a flexión, son las más abundantes de las estructuras metálicas y aparecen bien en compresión pura o mezcladas con flexión en:

- los pilares de los edificios

- las barras de las estructuras trianguladas

- los arcos, etc.

La Norma MV-103 o E.A.95 posee un coeficiente $\beta$ que, multiplicado a la longitud de la barra, nos da el valor de la longitud de pandeo, que será la medida que utilizaremos en los cálculos de dimensionamiento y diseño de la estructura.

Debido a que se han encontrado errores en algunos de los valores aproximados del coeficiente $\beta$ de la Norma $M V-103$ del año 1972 y que estos errores continúan en la actualización de 1995, E.A.95, en el artículo se estudiarán los casos concretos de las tablas 3.2, 3.3 y 3.4 de la Norma MV-103, que coincide con la E.A.95. 
Se han encontrado errores en los casos $1 b, 1 d, 1 e$ y $1 f$ de la tabla 3.2 donde se da un valor del coeficiente $s$ igual a:

$$
s=\frac{I}{b^{2}}\left(\frac{l}{A}+\frac{l}{A_{1}}\right) \leq 0,2, \text { cuando la ecuación correcta es: } s=\frac{I}{b^{2}}\left(\frac{1}{A}+\frac{1}{A_{1}}\right) \leq 0,2
$$

Además, las ecuaciones que dan el valor del coeficiente $\beta$ de los casos $1 e$ y $1 f$, están trastocadas, la del $1 e$ sería en realidad la del $l f \mathrm{y}$ al contrario.

En los estudios del doble y triple vano, debido a la no constancia de los momentos que se presentan en los de un vano, se han establecido unos valores concretos y, comparando los resultados, en el caso $2 a$ nuestro valor de $\beta$ para el pilar central y el de la $M V$ - 103 coinciden, mientras que para los pilares extremos hay una diferencia del $43 \%$. Es notable, pero no peligrosa, al estar del lado de la seguridad o ser más rígida. En el $2 b$ ocurre lo mismo.

En el caso $2 c$ para los pilares extremos coinciden las $\beta$, pero para el central existe una diferencia del $41 \%$. Es notable y más peligrosa debido a que no está en el lado de la seguridad o es menos rígida.

Comprobada también la tabla 3.3 se han encontrado diferencias de hasta un $20 \%$, pero siempre por el lado de la seguridad. Para la tabla 3.4 existen diferencias de un $18 \%$ pero, en este caso, no por el lado de la seguridad

Todos los estudios estarán dentro del régimen elástico, donde las tensiones permanecen por debajo del límite elástico o de proporcionalidad del material, siguiendo, éste, la Ley de Hooke:

$$
\varepsilon=\frac{\sigma}{\mathrm{E}}
$$

donde:

$$
\begin{aligned}
& \text { - } \varepsilon \text { es el alargamiento unitario } \\
& \text { - } \sigma \text { es la tensión en } \mathrm{kp} / \mathrm{cm}^{2} \\
& \text { - } E \text { es el módulo elástico en } \mathrm{kp} / \mathrm{cm}^{2}
\end{aligned}
$$

Dentro del régimen plástico, el material no responde a la Ley de Hooke. El valor de la razón do/d $\varepsilon$ puede considerarse como un módulo de elasticidad variable del material, que es función de $\sigma$ y lo designaremos por $\mathrm{E}_{\sigma}$.

En todos los casos se supondrá que los nudos del pórtico tienen libertad de giros y corrimientos dentro del plano, que están impedidos los corrimientos en dirección perpendicular al plano del pórtico y que la flexión se efectúa en uno de los dos planos principales de la barra.

En todos estos problemas se suponen siempre las deformaciones lo suficientemente pequeñas, como son en realidad, aun en el colapso de la pieza. Esto hace que la tangente, el seno y el ángulo sean aproximadamente iguales y podamos tomar uno $\mathrm{u}$ otro.

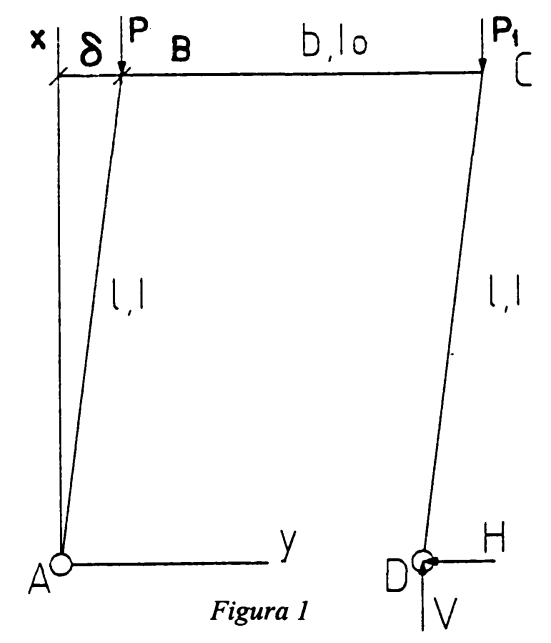

Vamos a solucionar el problema dentro del régimen elástico de una manera exacta, planteando la ecuación diferencial. Después se compararán gráficamente los resultados con la aproximación de la E.A. 95.

El caso de la figura 1 es el resuelto por Timoshenko en su libro "TEORÍA DE LA ESTABILIDAD ELÁSTICA". Pero la estructura es simétrica hasta en la carga.

"Cuando el marco no es simétrico o no está simétricamente cargado, el problema de determinar el valor crítico de la carga se hace más complicado puesto que se necesita considerar corrimientos y giros de las uniones superiores", nos dice y nos remite $a R$. $v$. Mises y J. Ratzersdorfer, $Z$. angew. Math. Mech., vol. 3, pag. 407, 1923. 
Ante esto, la suposición de que el momento en el nudo es $P \cdot y$, del que parte el citado autor, hay que demostrarlo resolviendo el caso hiperestático del pórtico en ese estado de deformación con su desplazamiento $\delta$. Resolviendo el caso encontramos que el momento en el nudo $B$ es constante y vale:

$$
M_{B}=M_{c}=\left(P+P_{1}\right) \frac{\delta}{2}
$$

Siguiendo la figura 1, examinemos este marco con barras verticales comprimidas y un dintel en condiciones de moverse lateralmente. El marco posee un eje vertical de simetría en cuanto a sus elementos, no en cuanto a la carga, coincidiendo con el caso a de la E.A. 95. El problema completo sería estudiarlo sin esta simetría, pero su estudio prácticamente no tiene interés, como lo demuestra el que la $E . A .95$ no lo incluya, pero el planteamiento es idéntico al que se realiza en este estudio. En este caso el momento en el nudo $B$ no sería $\left(\mathrm{P}+\mathrm{P}_{1}\right) \delta / 2$ y habría que estudiarlo aparte.

Comprobados todos los casos de la tabla 3.2, caso 1 , con las correcciones antes dichas, la coincidencia de ambas soluciones es muy aceptable. Por lo que, debido al espacio reducido de un artículo, sólo vamos a exponer, de los casos 1 , el $1 d$ y $l e$, una vez realizados los cambios, para demostrar el error de la Norma, aunque lo corrobora el Sr. Rodríguez-Avial Azcúnaga en su libro "CONSTRUCCIONES METÁLICAS", quinta edición (1963), pág. 631, en los casos $1 b, 1 d$. Los casos le, lf no aparecen, aunque el error, sólo por comparación, se puede ver.

De los casos 2 sólo tocaremos el $2 c$, al ser más peligroso, por no estar de lado de la seguridad.

De las tablas 3.3 y 3.4 , por el mismo motivo, expondremos el caso de estructuras sin recuadros arriostrados, o la tabla 3.4 .

\section{CASO 1d}

\begin{tabular}{|c|c|c|c|}
\hline CASO & FORMA DEL PÓRTICO & COEFICIENTE $\beta$ & $\begin{array}{l}\text { MAGNITUDES } \\
\text { AUXILIARES }\end{array}$ \\
\hline ld & 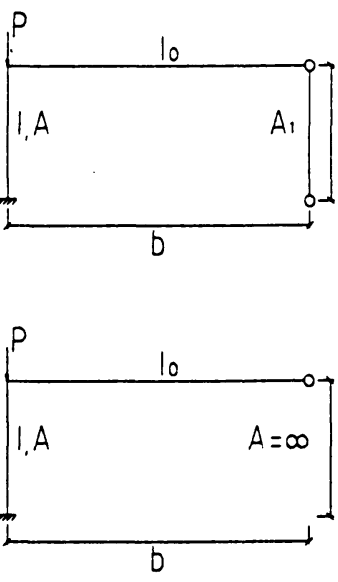 & $\sqrt{1+0,35(c+6 s)-0,017(c+6 s)^{2}}$ & $\begin{array}{l}c=\frac{2 I b}{I_{0} l} \leq 10 \\
s=\frac{I}{b^{2}}\left(\frac{1}{A}+\frac{1}{A_{1}}\right) \leq 0,2\end{array}$ \\
\hline
\end{tabular}

Como en el caso $1 b$, creemos que la fórmula dada por la Norma está equivocada y que el valor de la constante $s$ debería ser:

$$
s=\frac{I}{b^{2}}\left(\frac{1}{A}+\frac{1}{A_{1}}\right) \text { y no } s=\frac{I}{b^{2}}\left(\frac{l}{A}+\frac{l}{A_{1}}\right)
$$

Para los cálculos que siguen se ha utilizado el valor de $s$ que creemos correcto, ya que con el de la Norma las diferencias entre los valores de los coeficientes $\beta$ son demasiado grandes.

Suponemos el caso de un pórtico empotrado en una base y articulado en la otra. Cargado con una carga de compresión $P$ sobre el pilar empotrado. El dintel está unido rígidamente al pilar empotrado y mediante una articulación al pilar articulado. 
En este caso, la figura deformada será de la figura 2. Partiendo de la ecuación diferencial calcularemos el coeficiente de la estructura.

La ecuación diferencial es:

$$
E I \frac{d^{2} y}{d x^{2}}=P(\delta-y)-M
$$

donde: $E I$ es la rigidez de flexión, $P$ la fuerza axil, $\mathrm{y}=\mathrm{f}(\mathrm{x})$, la ecuación de la elástica.

hallamos la ecuación trascendente:

$$
\frac{\operatorname{tg} k l}{k l}=-\frac{b I}{3 l I_{0}}-\frac{I}{b^{2}}\left(\frac{1}{A}+\frac{1}{A_{1}}\right)
$$

o lo que es lo mismo:

$$
\frac{\operatorname{tg} k l}{k l}=-\frac{c+6 s}{6}
$$

Encontrado el valor de $k l$ podemos hallar $\beta$ : $\beta=\frac{\pi}{k l}$

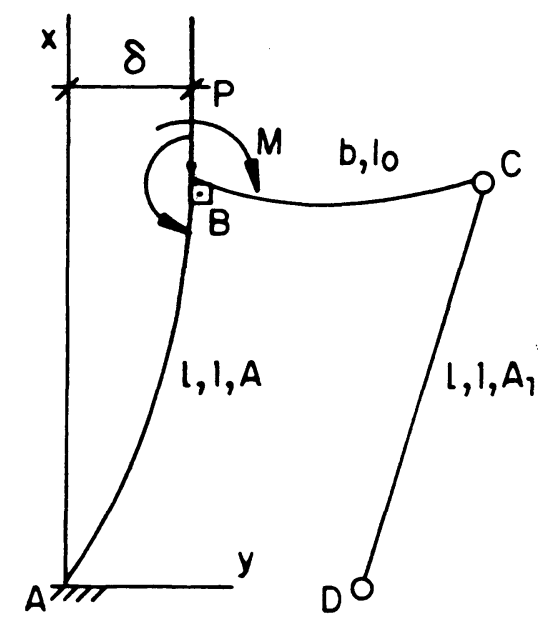

Figura 2

En las tablas y gráficas siguientes podemos comparar el valor de $\beta$ que hemos obtenido con la aproximación de la Norma. En la gráfica I se han tomado los siguientes valores: $b=600 \mathrm{~cm}, I=I_{0}=9.800 \mathrm{~cm}^{4}, A=A_{1}=69 \mathrm{~cm}^{2}$ y se ha variado la longitud $l$. En la gráfica II se ha tomado $l=b=600 \mathrm{~cm}, I=I_{I}=9.800 \mathrm{~cm}^{4}, A=69 \mathrm{~cm}^{2}$, pero se ha variado la sección del pilar derecho $A_{1}$.

TABLA I.

\begin{tabular}{|c|c|c|c||c|c|c|c||c|c|c|c|}
\hline$l$ & $k l$ & $\beta$ & $\beta_{103}$ & $l$ & $k l$ & $\beta$ & $\beta_{103}$ & $l$ & $k l$ & $\beta$ & $\beta_{103}$ \\
\hline 100 & 1,8365 & 1,7106 & 1,6588 & 400 & 2,2882 & 1,3729 & 1,3777 & 700 & 2,5168 & 1,2482 & 1,2455 \\
\hline 150 & 1,9395 & 1,6197 & 1,6469 & 450 & 2,3364 & 1,3445 & 1,3467 & 750 & 2,5441 & 1,2348 & 1,2320 \\
\hline 200 & 2,0284 & 1,5487 & 1,5775 & 500 & 2,3798 & 1,3201 & 1,3203 & 800 & 2,5692 & 1,2227 & 1,2199 \\
\hline 250 & 2,1060 & 1,4917 & 1,5130 & 550 & 2,4190 & 1,2986 & 1,2977 & 850 & 2,5923 & 1,2118 & 1,2090 \\
\hline 300 & 2,1741 & 1,4449 & 1,4591 & 600 & 2,4546 & 1,2798 & 1,2780 & 900 & 2,6138 & 1,2019 & 1,1991 \\
\hline 350 & 2,2345 & 1,4059 & 1,4146 & 650 & 2,4871 & 1,2631 & 1,2608 & & & & \\
\hline
\end{tabular}




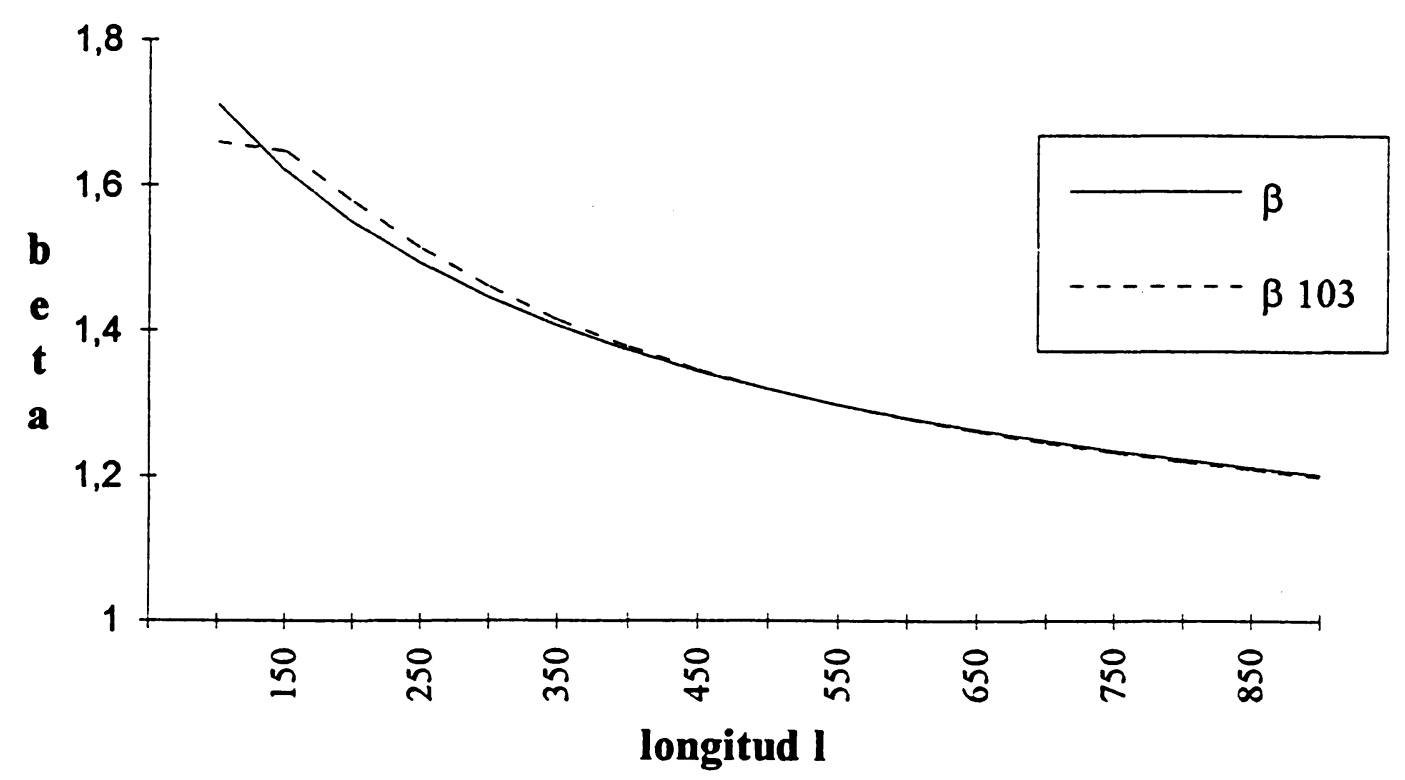

Gráfica $I$

TABLA II.

\begin{tabular}{|c|c|c|c|c||c|c|c|c|c|}
\hline$I P N$ & $A_{1}$ & $k l$ & $\beta$ & $\beta_{103}$ & $I P N$ & $A_{1}$ & $k l$ & $\beta$ & $\beta_{103}$ \\
\hline 80 & 7,57 & 2,4508 & 1,2818 & 1,2801 & 320 & 77,7 & 2,4547 & 1,2798 & 1,2780 \\
\hline 140 & 18,2 & 2,4533 & 1,2805 & 1,2787 & 380 & 107 & 2,4548 & 1,2797 & 1,2779 \\
\hline 200 & 33,4 & 2,4541 & 1,2801 & 1,2783 & 450 & 147 & 2,4549 & 1,2797 & 1,2779 \\
\hline 260 & 53,3 & 2,4545 & 1,2799 & 1,2781 & 500 & 179 & 2,4550 & 1,2796 & 1,2778 \\
\hline
\end{tabular}

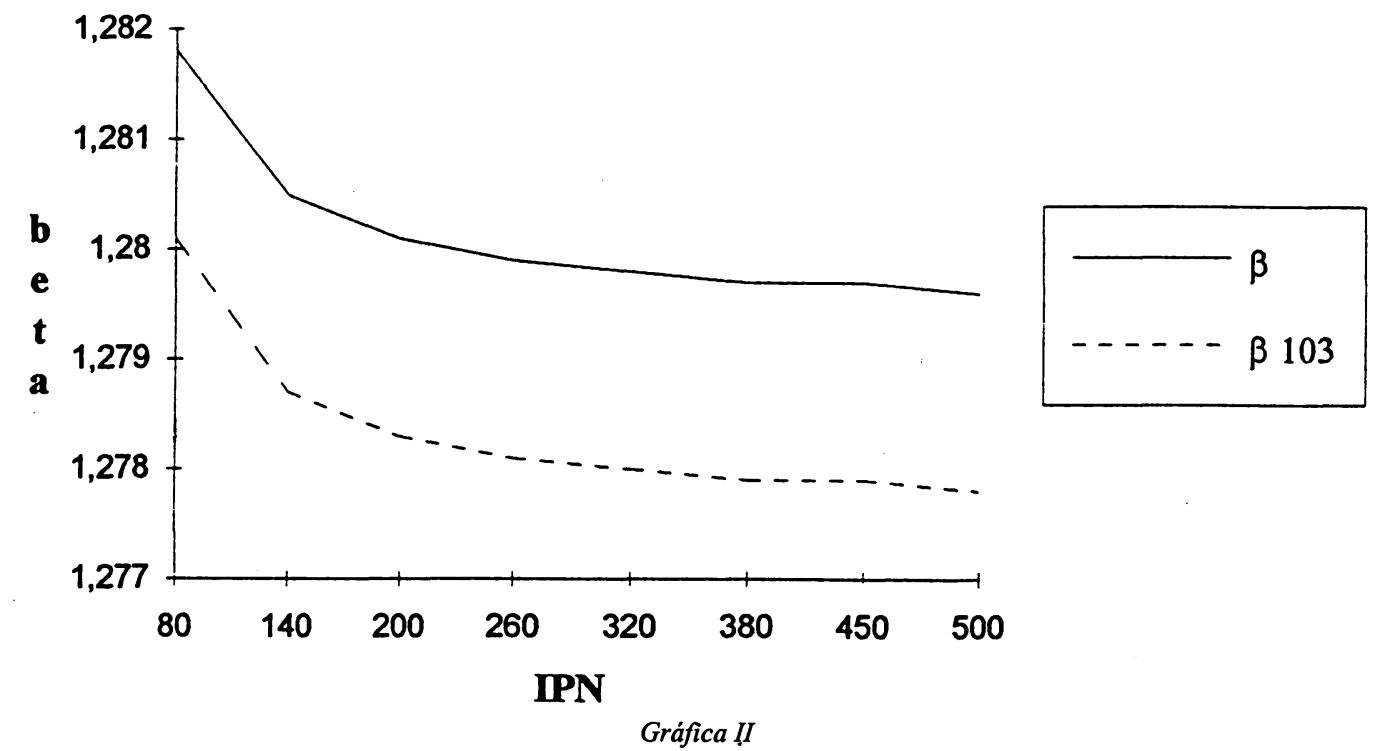




\section{CASO 1e}

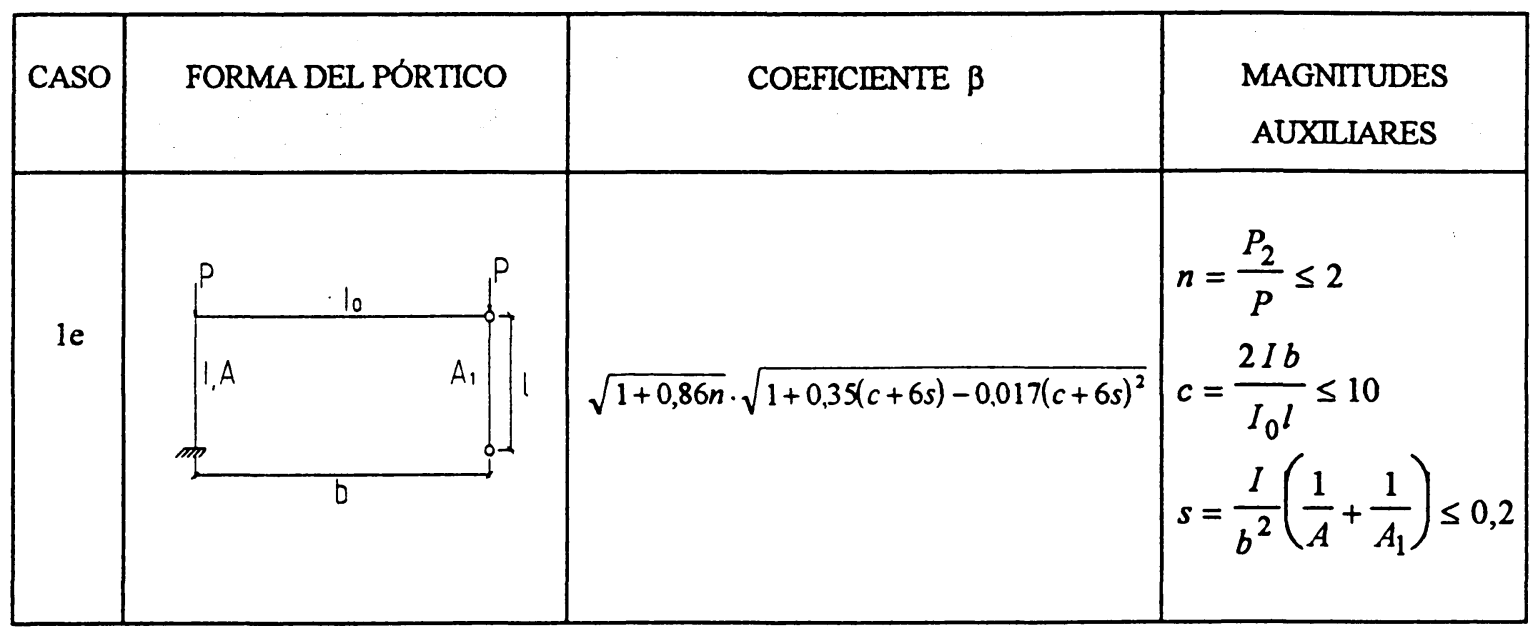

La Norma $M V-103$ y la $E . A .95$ dan un valor de la constante: $\beta=\sqrt{1+0,96 n} \sqrt{4+1,4(c+6 s)+0,02(c+6 s)^{2}}$

que creemos equivocado,ya que se han comparado los valores del coeficiente $\beta$ hallados con la aproximación que da la Norma y son muy diferentes. Vemos que este caso es similar al caso $l c$ donde el extremo del pilar izquierdo está empotrado en su extremo inferior, por lo que la ecuación de la constante $\beta$ también debería ser similar. Al estudiar el caso $1 f$ se ha comprobado que también existían diferencias entre los valores de $\beta$ y que la ecuación que presenta la Norma debería ser similar a la del caso $1 a$, ya que las estructuras son muy similares al estar el pilar izquierdo articulado. De todo esto se ha deducido que las ecuaciones de los casos le y $1 f$ están intercambiadas.

Por lo tanto, la ecuación correcta en el caso le será : $\beta=\sqrt{1+0,86 n} \sqrt{1+0,35(c+6 s)-0,017(c+6 s)^{2}}$

en la que podemos ver que la segunda raíz es similar a la del caso $1 c$ y, por lo tanto, será la ecuación utilizada en los cálculos.

Es un caso similar al caso $1 D$, pero aplicando además otra carga de compresión $P_{2}$ en el pilar $C D$. La deformada será la de la figura 3.

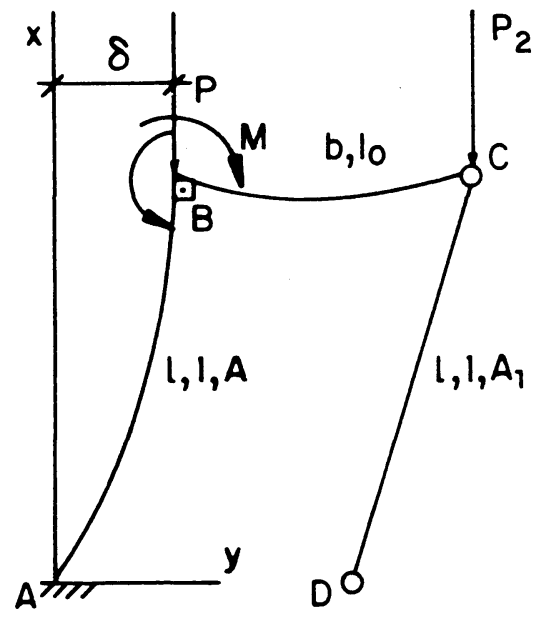

Figura 3
Solucionando la ecuación diferencial:

$$
E I \frac{d^{2} y}{d x^{2}}=\left(P+P_{2}\right)(\delta-y)-M
$$

obtenemos la ecuación trascendente cuya incógnita es $\mathrm{kl}$ :

$$
\frac{\operatorname{tg} k l}{k l}=-\frac{b I}{3 l I_{0}}-\frac{I}{b^{2}}\left(\frac{1}{A}+\frac{1}{A_{1}}\right)
$$

o lo que es lo mismo: $\frac{\operatorname{tg} k l}{k l}=-\frac{c+6 s}{6}$

Hallamos el valor de $\beta: \beta=\sqrt{\frac{\pi^{2}(1+n)}{(k l)^{2}}}$

Para la gráfica I, se han tomado los siguientes valores de las constantes: $l=b=600 \mathrm{~cm}, I=I_{o}=9.800 \mathrm{~cm}^{4}$, $A=A_{1}=69 \mathrm{~cm}^{2}$ y se ha variado $n$. En la gráfica II se han mantenido los mismos valores, pero se ha fijado $n=1$ y se ha variado la longitud $l$. 
TABLA I.

\begin{tabular}{|c|c|c|c|c|c|c|c|c|c|c|c|}
\hline$n$ & $k l$ & $\beta$ & $\beta_{103}$ & $n$ & $k l$ & $\beta$ & $\beta_{103}$ & $n$ & $k l$ & $\beta$ & $\beta_{103}$ \\
\hline 0 & \multirow{5}{*}{2,4546} & 1,2798 & 1,2780 & 1,0 & \multirow{4}{*}{2,4546} & 1,8099 & 1,7430 & 1,8 & \multirow{4}{*}{2,4546} & 2,1415 & 2,0400 \\
\hline 0,2 & & 1,4019 & 1,3836 & 1,2 & & 1,8983 & 1,8218 & 2 & & 2,2167 & 2,1077 \\
\hline 0,4 & & 1,5143 & 1,4816 & 1,4 & & 1,9827 & 1,8973 & 2,2 & & 2,2894 & 2,1734 \\
\hline 0,6 & & 1,6188 & 1,5736 & 1,6 & & 2,0637 & 1,9700 & 2,4 & & 2,3599 & 2,2371 \\
\hline 0,8 & & 1,7170 & 1,6604 & & & & & & & & \\
\hline
\end{tabular}

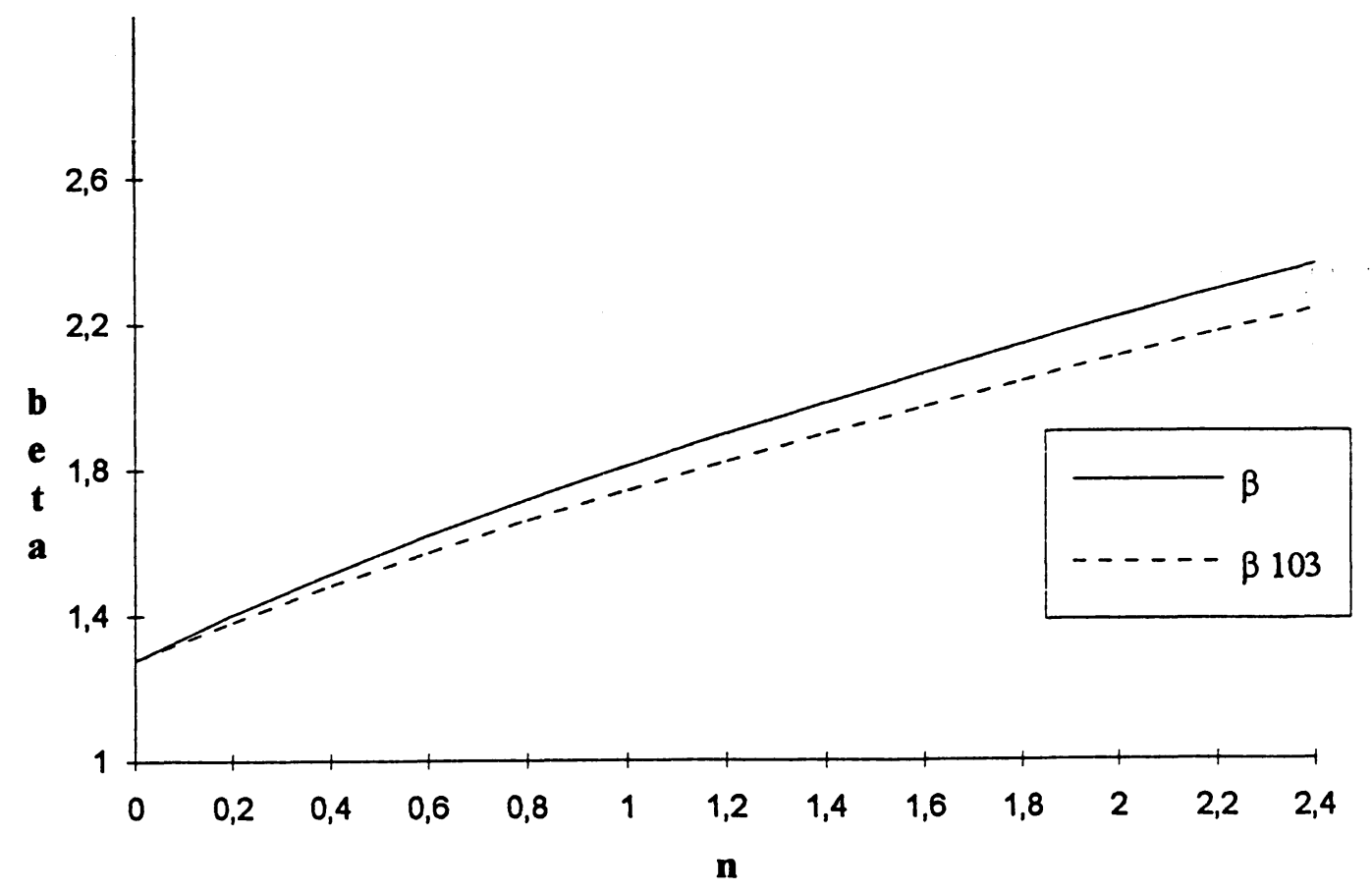

Gráfica I 
TABLA II

\begin{tabular}{|c|c|c|c||c|c|c|c||c|c|c|c|}
\hline$l$ & $k l$ & $\beta$ & $\beta_{103}$ & $l$ & $k l$ & $\beta$ & $\beta_{103}$ & $l$ & $k l$ & $\beta$ & $\beta_{103}$ \\
\hline 100 & 1,8365 & 2,4192 & 2,2623 & 350 & 2,2345 & 1,9883 & 1,9293 & 600 & 2,4546 & 1,8099 & 1,7430 \\
\hline 150 & 1,9396 & 2,2906 & 2,2461 & 400 & 2,2883 & 1,9415 & 1,8790 & 650 & 2,4871 & 1,7863 & 1,7195 \\
\hline 200 & 2,0285 & 2,1902 & 2,1515 & 450 & 2,3364 & 1,9015 & 1,8367 & 700 & 2,5168 & 1,7652 & 1,6987 \\
\hline 250 & 2,1060 & 2,1096 & 2,0635 & 500 & 2,3798 & 1,8669 & 1,8007 & 750 & 2,5441 & 1,7463 & 1,6802 \\
\hline 300 & 2,1741 & 2,0435 & 1,9899 & 550 & 2,4190 & 1,8366 & 1,7698 & 800 & 2,5692 & 1,7292 & 1,6637 \\
\hline
\end{tabular}

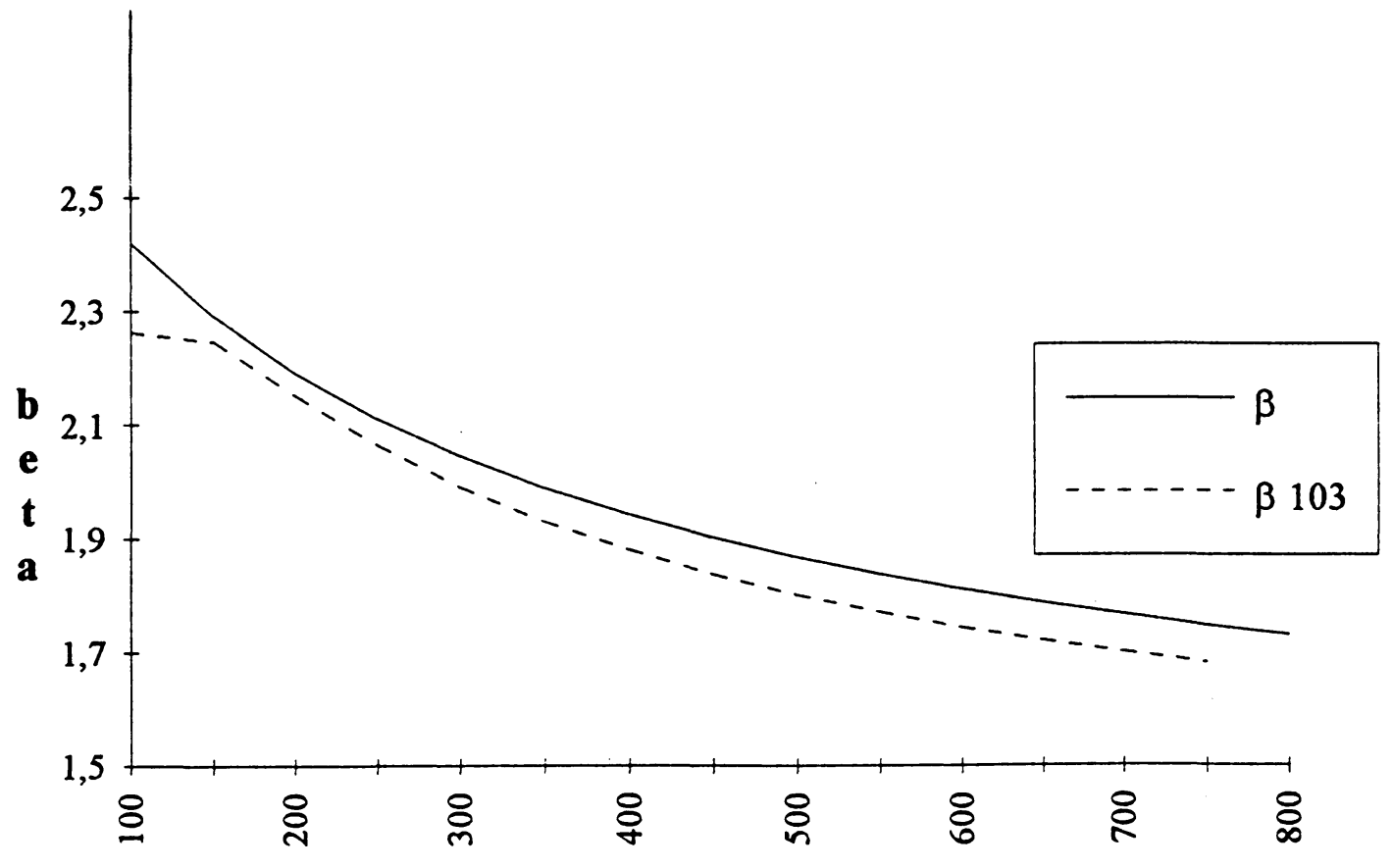

longitud I

Gráfica II 
CASO 2c

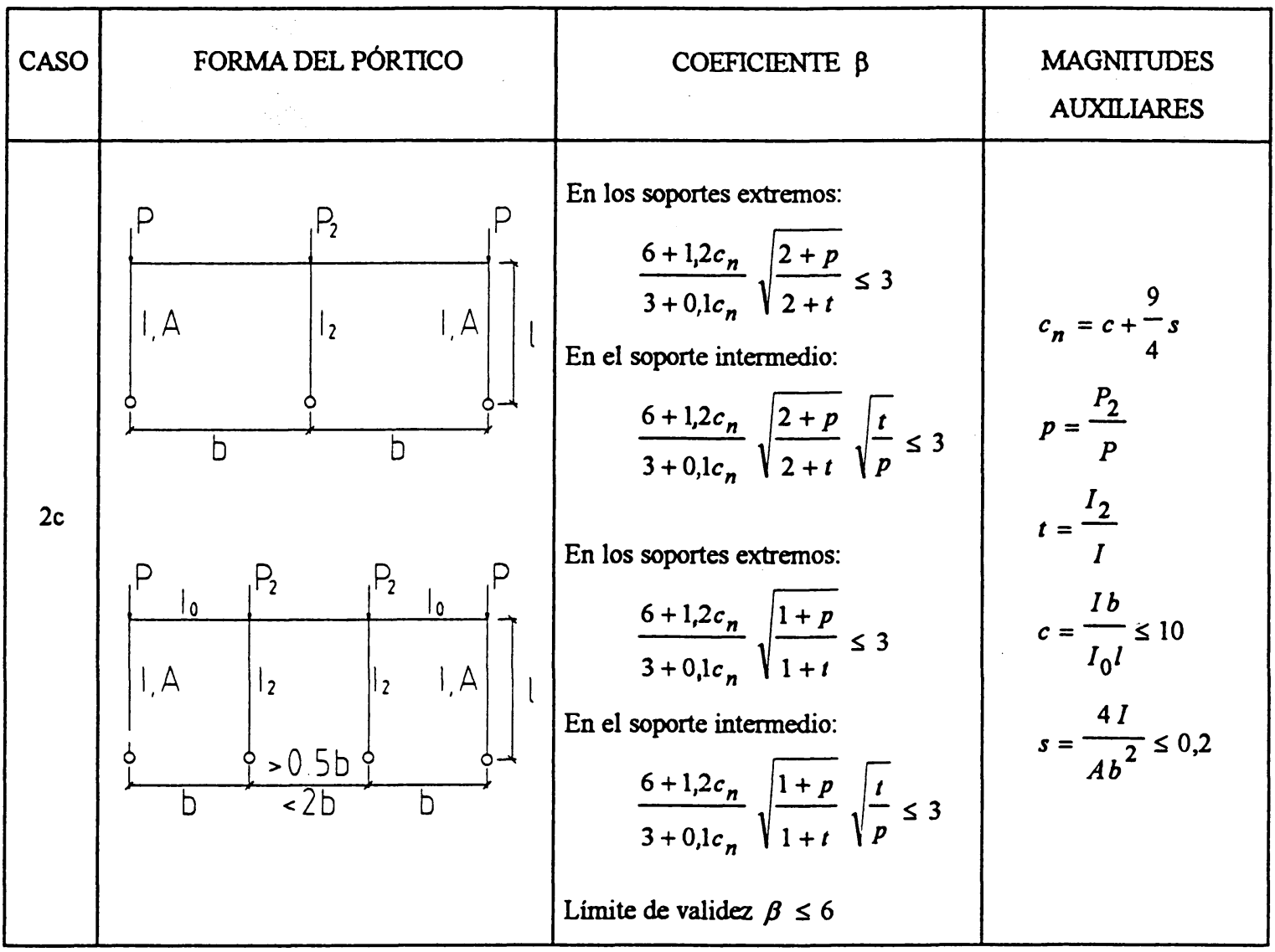

Se ha comprobado que los momentos no son constantes, por lo que tenemos que estudiar un caso concreto, con unos valores reales. Estudiaremos la figura 4, donde supondremos el arriostramiento en sentido perpendicular al plano del dibujo y de acero A-42.

$\mathrm{Al}$ estar los pilares comprimidos la rigidez y el coeficiente de transmisión variarán en función del parámetro $\rho=\frac{\mathrm{P}}{\mathrm{P}_{\mathrm{er}}}$, mientras que en las barras horizontales, al no haber compresión, será $\frac{4 E I}{1}$ la rigidez y 0,5 el coeficiente de transmisión.

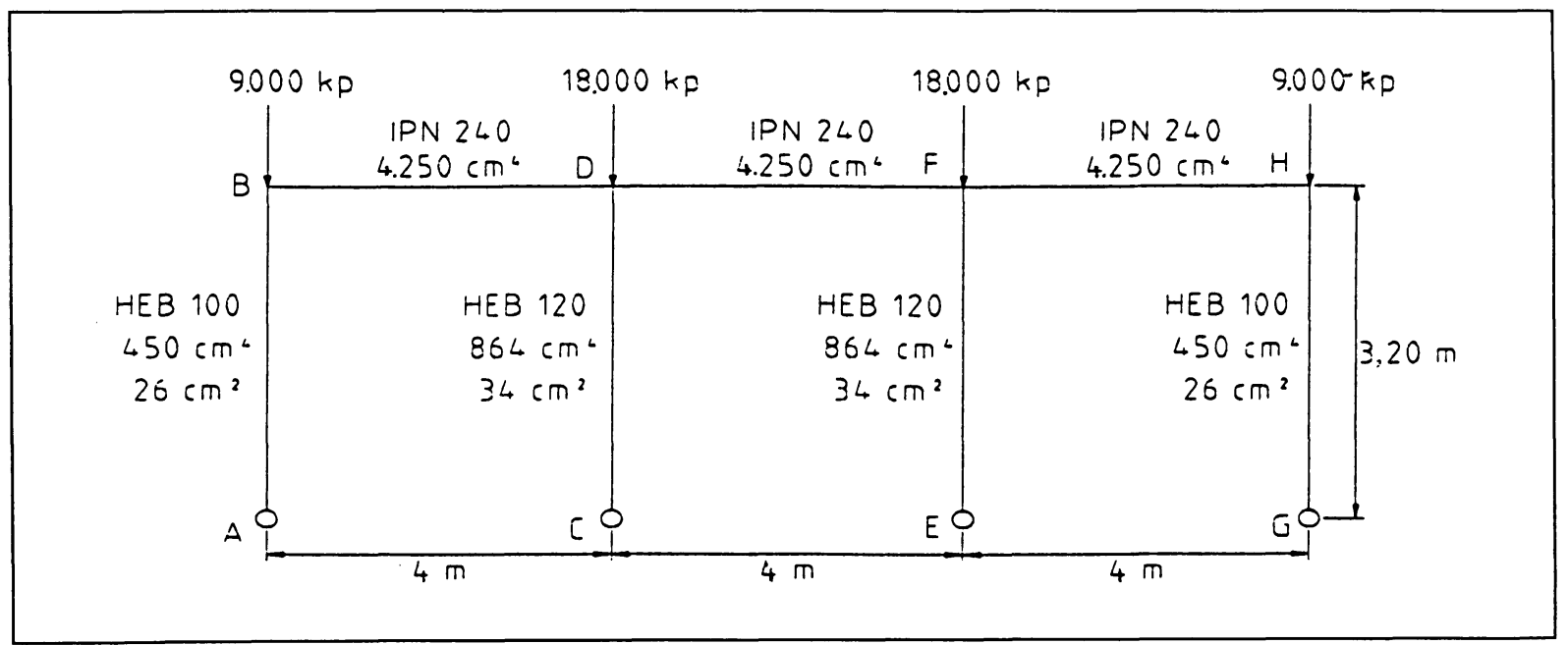


La ecuación que nos da el valor del coeficiente de transmisión para los pilares es: $r=\frac{k l-\operatorname{sen} k l}{\operatorname{sen} k l-k l \cos k l}$ donde $k=\sqrt{\frac{P}{E I}}$

también comprobamos que la rigidez para los pilares es:

$$
K=\frac{\frac{k l}{2}(1-k l \cot k l)}{\operatorname{tg} \frac{k l}{2}-\frac{k l}{2}} \frac{E I}{l}
$$

\section{Cálculo del coeficiente $\beta$ para los pilares intermedios}

Momentos en los nudos:

$$
\begin{gathered}
2\left(P+P_{2}\right) \frac{\delta}{320}=\frac{2596,102}{320} x \Rightarrow \quad x=2\left(P+P_{2}\right) \frac{\delta}{2596,102} \\
M_{B A}=M_{H G}=M_{B D}=M_{H F}=444,094 \cdot 2\left(P+P_{2}\right) \frac{\delta}{2596,102}=0,342\left(P+P_{2}\right) \delta \\
M_{D C}=M_{F E}=853,866 \cdot 2\left(P+P_{2}\right) \frac{\delta}{2596,102}=0,658\left(P+P_{2}\right) \delta \\
M_{D B}=M_{F G}=432,692 \cdot 2\left(P+P_{2}\right) \frac{\delta}{2596,102}=0,333\left(P+P_{2}\right) \delta \\
M_{D E}=M_{F D}=421,174 \cdot 2\left(P+P_{2}\right) \frac{\delta}{2596,102}=0,325\left(P+P_{2}\right) \delta
\end{gathered}
$$

Planteamiento de la ecuación diferencial: $E I \frac{d^{2} y}{d x^{2}}=-0,342\left(P+P_{2}\right) y$ siendo $\mathrm{p}=\frac{\mathrm{P}_{2}}{\mathrm{P}}$ y Q $=0,342 \mathrm{P}(1+\mathrm{p})$ la ecuación diferencial nos queda: $E I \frac{d^{2} y}{d x^{2}}=-Q y$

Ecuación trascendente: $k l \operatorname{tg} k l=\frac{2,052}{0,351 \frac{b I}{l I_{0}}+0,009 \frac{4 I}{A b^{2}}} \quad \Rightarrow \quad k l \operatorname{tg} k l=\frac{2,052}{0,351 c+0,009 s}$

Ecuación del coeficiente $\beta: \beta=\sqrt{\frac{\pi^{2} 0,342(1+p)}{(k l)^{2}}}$ 
Comparamos el valor del coeficiente $\beta$ que hemos hallado con el valor que da la Norma y obtenemos el mismo resultado:

$$
\begin{aligned}
& k l=1,536 \\
& \beta=2,0717 \\
& \beta_{103}=2,0720
\end{aligned}
$$

\section{Cálculo del coeficiente $\beta$ para los pilares intermedios}

Planteamiento de la ecuación diferencial: $E I_{2} \frac{d^{2} y}{d x^{2}}=-0,658\left(P+P_{2}\right) y$

siendo $\mathrm{p}=\frac{\mathrm{P}_{2}}{\mathrm{P}}$ y $\mathrm{Q}=0,658 \mathrm{P}(1+\mathrm{p})$ la ecuación diferencial nos queda: $E I_{2} \frac{d^{2} y}{d x^{2}}=-Q y$

Ecuación trascendente:

$$
k l \operatorname{tg} k l=\frac{3,948}{0,324 \frac{b I}{l I_{0}} \frac{I_{2}}{I}+0,675 \frac{4 I}{A b^{2}} \frac{I_{2}}{I}} \Rightarrow k l \operatorname{tg} k l=\frac{3,948}{0,324 c t+0,675 s t}
$$

Ecuación del coeficiente $\beta: \beta=\sqrt{\frac{\pi^{2} 0,325(1+p)}{(k l)^{2}}}$

Comparamos el valor del coeficiente $\beta$ que hemos hallado con el valor que da la Norma y obtenemos un resultado bastante diferente y además nuestro caso, como pasaba en el caso del doble pórtico, no va por el lado de la seguridad:

$$
\begin{aligned}
& k l=1,5385 \\
& \beta=2,8689 \\
& \beta_{103}=2,0302
\end{aligned}
$$

\section{COEFICIENTE $\beta$ PARA PILARES DE ESTRUCTURAS DE VARIOS PISOS SIN RECUADROS ARRIOSTRADOS.}

Hemos visto que los pórticos simples se pueden resolver fácilmente haciendo uso de las ecuaciones diferenciales. Para pórticos más complicados, como son las estructuras de edificación, es necesario recurrir a métodos matriciales que exigen conocer las rigideces de las barras y sus coeficientes de transmisión.

Por efecto de una compresión tanto la rigidez como el coeficiente de transmisión pueden verse afectados, convirtiéndose en funciones de esta misma compresión.

Para estudiar la rigidez y el factor de transmisión consideraremos la barra de la figura 5, que está empotrada en el extremo $A$, articulada en el extremo $B$ y sometida a una compresión $P$. Se le aplica en el extremo articulado un momento $M$ por efecto del cual, el nudo $B$ gira un ángulo $\theta_{B}$ y en el empotramiento $A$ aparece un momento inducido $m$. 


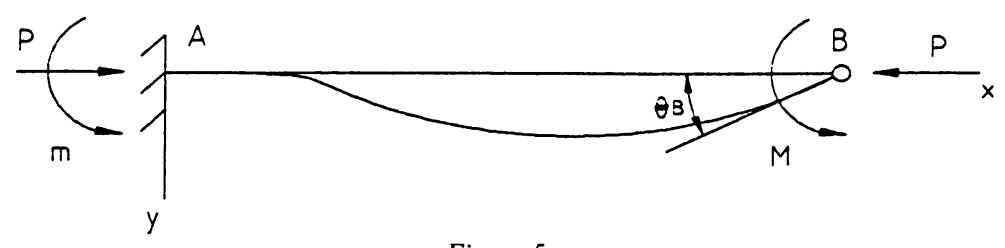

Figura 5

Llamamos rigidez del nudo $B$ al momento necesario para producir un giro de un radián en el extremo $B$ (suponiendo que no se rebase el límite de proporcionalidad del material y que las deformaciones pudiesen seguir considerándose pequeñas). Su valor será:

$$
K=\frac{M}{\theta_{B}}
$$

Llamamos factor de transmisión del nudo B al $\boldsymbol{A}$ a la relación entre el momento reacción $m$ que aparece en el extremo empotrado $A$ y el momento $M$ aplicado en el extremo $B$,

$$
r=\frac{m}{M}
$$

Se demuestra que:

$$
m=M \frac{k l-\operatorname{sen} k l}{\operatorname{sen} k l-k l \cos k l} \quad ; \quad r=\frac{k l-\operatorname{sen} k l}{\operatorname{sen} k l-k l \cos k l} \quad ; \quad k=S \frac{E I}{l}
$$

donde $\quad k^{2}=\frac{P}{E I} \quad$ y $\quad S=\frac{\frac{k l}{2}(1-k l \cot k l)}{\operatorname{tg} \frac{k l}{2}-\frac{k l}{2}}$

Cuando la viga no está comprimida, $P=0$, o lo que es lo mismo $k l=0$, el valor límite de $r$ y de $S$ es: $\mathrm{r}=0,5$ y $\mathrm{S}=4$

En el estudio de la estabilidad de las estructuras de varios pisos con desplazamiento lateral no coartado, la fuerza cortante en los extremos de un pilar debe ser cero en la posición de equilibrio.

Consideramos la viga de la figura 6:

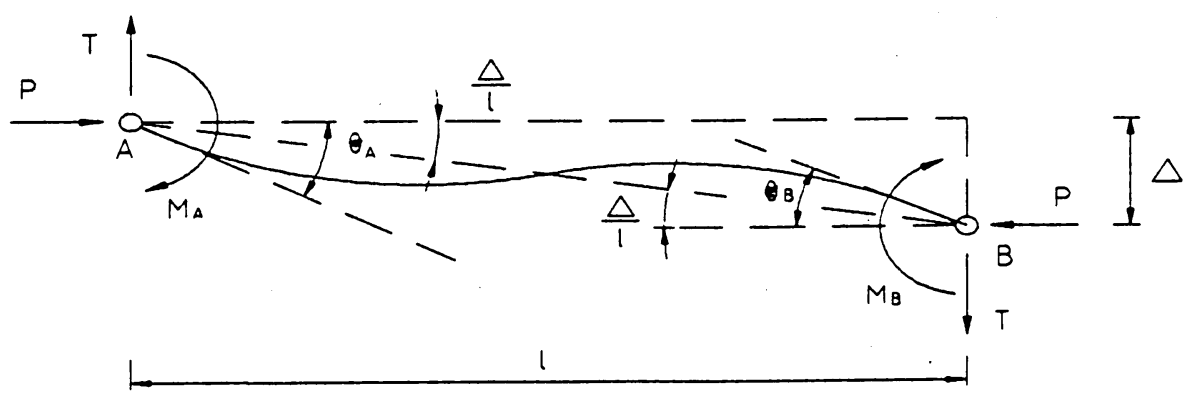

Figura 6 
Resolviendo por la estática tenemos las siguientes ecuaciones:

$$
\begin{aligned}
& M_{A}=\left[\left[S-S(r+1) \frac{m}{2}\right] \theta_{A}+\left[S r-S(r+1) \frac{m}{2}\right] \theta_{B}\right] R \\
& M_{B}=\left[\left[S r-S(r+1) \frac{m}{2}\right] \theta_{A}+\left[S-S(r+1) \frac{m}{2}\right] \theta_{B}\right] R
\end{aligned}
$$

donde $S$ y $r$ están definidas en el apartado anterior y $m$ y $R$ son:

$$
m=\frac{\Delta}{l} \frac{\left(\theta_{A}+\theta_{B}\right)}{2} \quad R=\frac{E l}{l}
$$

Para estudiar los casos que presenta la tabla 3.4 de la $M V-103$ (coeficiente $\beta$ para pilares de estructuras sin recuadros arriostrados) nos fijaremos en la estructura de la figura 7. Consideramos que se alcanza la carga crítica de una estructura cuando se alcanza la de uno de sus miembros. En dicha figura, los elementos comprimidos son los pilares. Conociendo la de cada pilar, la mínima nos dará la carga crítica de la estructura.

Para estudiar la estructura de la figura 7 se determina la carga crítica del pilar $A B$, asumiendo las siguientes simplificaciones:

1. Sólo se considera la influencia de las vigas que concurren en los extremos $A$ y $B$ del pilar.

2. Se acepta que los nudos $E$ y $F$ estén fijos y empotrados.

3. Se acepta que los nudos $C$ y $D$ estén empotrados,pero que puedan desplazarse libremente, lo que indica que el cortante en el nudo $A$ del pilar debe ser cero.

Como en el caso de una estructura con recuadros arriostrados, llamaremos:

$$
R_{P}=\frac{E I_{P}}{l} ; \quad R_{A C}=\frac{E I_{A C}}{l_{A C}} ; \quad R_{A D}=\frac{E I_{A D}}{l_{A D}} ; \quad R_{B E}=\frac{E I_{B E}}{l_{B E}} ; \quad R_{B F}=\frac{E I_{B F}}{l_{B F}}
$$

donde todos los parámetros son valores conocidos.

Bajo la acción de una carga de compresión $P$ aplicada en los nudos $A$ y $B$, aparecerán unos giros $\theta_{A}$ y $\theta_{B}$ creando la deformada de la figura 8.

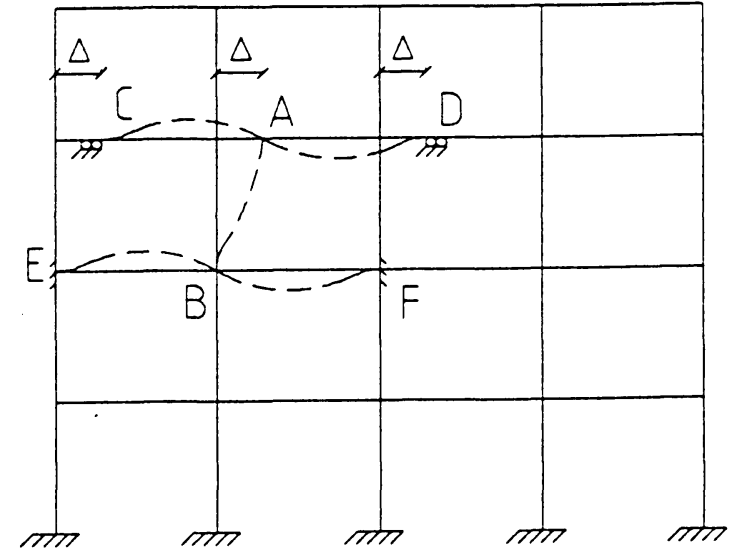

Figura 7

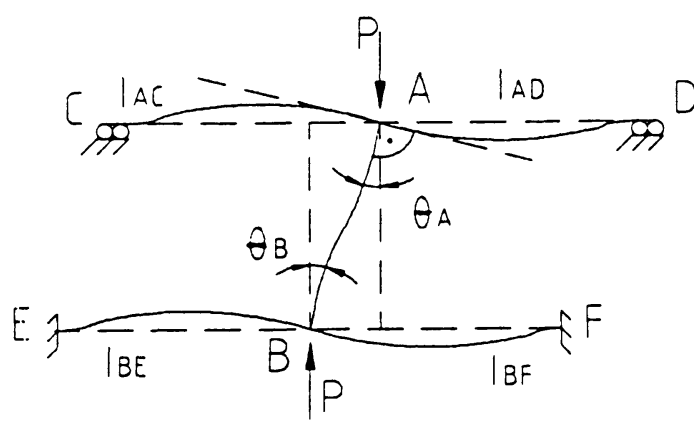

Figura 8 
Para valores de $P>P_{c r}$ aparecerán en los nudos $A$ y $B$ unos momentos $M_{A}$ y $M_{B}$ en equilibrio con la deformada. Como los extremos $C$ y $D$ pueden desplazarse, tenemos un equilibrio con cortante nulo, por lo que las ecuaciones de equilibrio de la barra $A B$ son:

$$
\left.\begin{array}{l}
M_{A}=\left(4 R_{A C}+4 R_{A D}\right) \theta_{A}+\left[\left[S-S(r+1) \frac{m}{2}\right] \theta_{A}+\left[S r-S(r+1) \frac{m}{2}\right] \theta_{B}\right] R_{P} \\
M_{B}=\left(4 R_{B E}+4 R_{B E}\right) \theta_{B}+\left[\left[S r-S(r+1) \frac{m}{2}\right] \theta_{A}+\left[S-S(r+1) \frac{m}{2}\right] \theta_{B}\right] R_{P}
\end{array}\right\}
$$

donde: $\left(4 R_{A C}+4 R_{A D}\right) \theta_{A} \quad$ es el momento absorbido por las barras $A C$ y $A D$.

$\left[\left[S-S(r+1) \frac{m}{2}\right] \theta_{A}+\left[S r-S(r+1) \frac{m}{2}\right] \theta_{B}\right] R_{P} \quad$ es el momento absorbido por la barra $A B$.

Para $P=P_{c r}$ el pilar $A B$ permanece en equilibrio,por lo que los momentos $M_{A}$ y $M_{B}$ serán cero. Para que exista equilibrio indiferente $\operatorname{con} \theta_{A} \neq 0$ y $\theta_{B} \neq 0$ el determinante de las ecuaciones de los momentos ha de ser cero. Resolviendo el determinante encontramos la ecuación trascendente siguiente:

$$
\left[\frac{\frac{k l}{2}[1-k l \cot k l]}{\operatorname{tg} \frac{k l}{2}-\frac{k l}{2}}-\frac{\left[\frac{\frac{k l}{2}[1-k l \cot k l]}{\operatorname{tg} \frac{k l}{2}-\frac{k l}{2}}\left(\frac{k l-\operatorname{sen} k l}{\operatorname{sen} k l-k l \cos k l}+1\right)\right]^{p}}{2 \frac{\frac{k l}{2}[1-k l \cot k l]}{\operatorname{tg} \frac{k l}{2}-\frac{k l}{2}}\left(\frac{k l-\operatorname{sen} k l}{\operatorname{sen} k l-k l \cos k l}+1\right)-(k l)^{2}}+\frac{4}{R_{A}}-4\right]
$$$$
\left[\frac{\frac{k l}{2}[1-k l \cot k l]}{\operatorname{tg} \frac{k l}{2}-\frac{k l}{2}}-\frac{\left[\frac{\frac{k l}{2}[1-k l \cot k l]}{\operatorname{tg} \frac{k l}{2}-\frac{k l}{2}}\left(\frac{k l-\operatorname{sen} k l}{\operatorname{sen} k l-k l \cos k l}+1\right)\right]^{2}}{2 \frac{\frac{k l}{2}[1-k l \cot k l]}{\operatorname{tg} \frac{k l}{2}-\frac{k l}{2}}\left(\frac{k l-\operatorname{sen} k l}{\operatorname{sen} k l-k l \cos k l}+1\right)-(k l)^{2}}+\frac{4}{R_{B}}-4\right]-
$$$$
-\left[\frac{\frac{k l}{2}[1-k l \cot k l]}{\operatorname{tg} \frac{k l}{2}-\frac{k l}{2}} \frac{k l-\operatorname{sen} k l}{\operatorname{sen} k l-k l \cos k l}-\left.\frac{\left[\frac{\frac{k l}{2}[1-k l \cot k l]}{\operatorname{tg} \frac{k l}{2}-\frac{k l}{2}}\left(\frac{k l-\operatorname{sen} k l}{\operatorname{sen} k l-k l \cos k l}+1\right)\right]^{2}}{2 \frac{\frac{k l}{2}[1-k l \cot k l]}{\operatorname{tg} \frac{k l}{2}-\frac{k l}{2}}\left(\frac{k l-\operatorname{sen} k l}{\operatorname{sen} k l-k l \cos k l}+1\right)-(k l)^{2}}\right|^{2}=0\right.
$$ 
Una vez obtenido el valor mínimo de $k l$, podemos obtener el valor de $P_{c r}$ y, por lo tanto, del coeficiente $\beta ; \beta=\frac{\pi}{k l}$

La Norma $M V-103$ nos da una ecuación aproximada del coeficiente $\beta$ en función del grado de empotramiento $k$ del pilar,

$$
k=\frac{\frac{I_{V}}{l_{V}}+\frac{I_{W}}{l_{W}}}{\frac{I}{l}+\frac{I_{P}}{l_{P}}+\frac{I_{V}}{l_{V}}+\frac{I_{W}}{l_{W}}}
$$

En nuestro caso tenemos:

$$
\begin{gathered}
k_{A}=\frac{\frac{I_{A C}}{l_{A C}}+\frac{I_{A D}}{l_{A D}}}{\frac{I_{A B}}{l_{A B}}+\frac{I_{A^{\prime}}}{l_{A^{\prime}}}+\frac{I_{A C}}{l_{A C}}+\frac{I_{A D}}{l_{A D}}} \quad k_{B}=\frac{\frac{I_{B E}}{l_{B E}}+\frac{I_{B F}}{l_{B F}}}{l_{A B}+\frac{I_{B B^{\prime}}}{l_{B B^{\prime}}}+\frac{I_{B E}}{l_{B E}}+\frac{I_{B F}}{l_{B F}}} \\
\beta=\sqrt{\frac{1,6+2,4\left(k_{A}+k_{B}\right)+1,1 k_{A} k_{B}}{\left(k_{A}+k_{B}\right)+5,5 k_{A} k_{B}}}
\end{gathered}
$$

A continuación se han representado, en unas gráficas, la variación del coeficiente $\beta$ según los momentos de inercia y las longitudes de las distintas barras de la estructura. Se ha representado el coeficiente $\beta$ según la expresión dada por la Norma $M V-103$ y el coeficiente $\beta$, calculado según la ecuación:

$$
\beta=\frac{\pi}{k l}
$$

TABLA I.

\begin{tabular}{|c|c|c|c|c|c|c|c|c|c|c|}
\hline$k_{A}$ & \multicolumn{10}{|c|}{0.0} \\
\hline$k_{B}$ & 0,0 & 0,1 & 0,2 & 0,3 & 0,4 & 0,5 & 0,6 & 0,7 & 0,8 & 0,9 \\
\hline$k l$ & - & 0,6211 & 0,8603 & 1,0298 & 1,1603 & 1,2645 & 1,3495 & 1,4198 & 1,4786 & 1,5283 \\
\hline$\beta$ & - & 5,0580 & 3,6515 & 3,0505 & 2,7074 & 2,4842 & 2,3278 & 2,2126 & 2,1246 & 2,0555 \\
\hline$\beta_{103}$ & - & 4,2895 & 3,2249 & 2,7808 & 2,5298 & 2,3664 & 2,2509 & 2,1646 & 2,0976 & 2,0439 \\
\hline
\end{tabular}

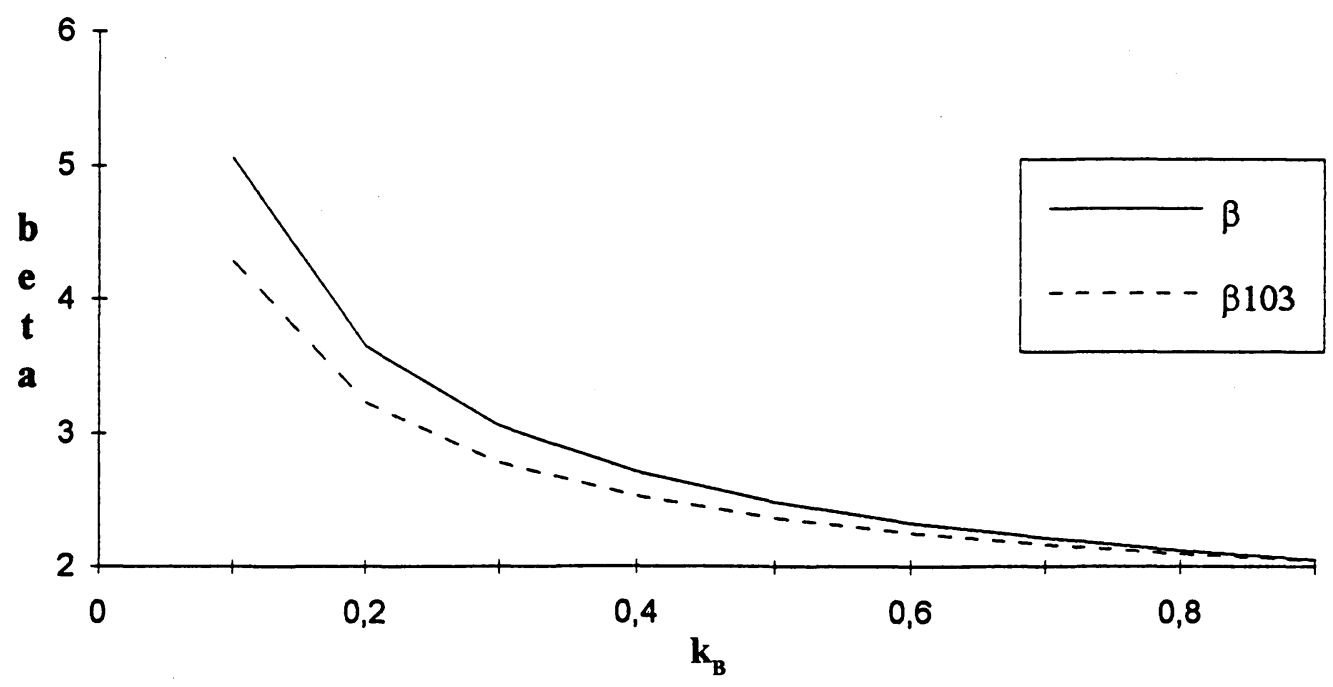

Gráfica $I\left(k_{a}=0\right)$ 
TABLA II.

\begin{tabular}{|c|c|c|c|c|c|c|c|c|c|c|}
\hline$k_{A}$ & \multicolumn{10}{|c|}{0,5} \\
\hline$k_{B}$ & 0,0 & 0,1 & 0,2 & 0,3 & 0,4 & 0,5 & 0,6 & 0,7 & 0,8 & 0,9 \\
\hline$k l$ & 1,2646 & 1,5000 & 1,7004 & 1,8737 & 2,0239 & 2,1537 & 2,2652 & 2,3606 & 2,4420 & 2,5113 \\
\hline$\beta$ & 2,4842 & 2,0943 & 1,8475 & 1,6766 & 1,5522 & 1,4586 & 1,3868 & 1,3307 & 1,2864 & 1,2509 \\
\hline$\beta_{103}$ & 2,3664 & 1,8807 & 1,6468 & 1,5059 & 1,4106 & 1,3416 & 1,2891 & 1,2477 & 1,2142 & 1,1865 \\
\hline
\end{tabular}

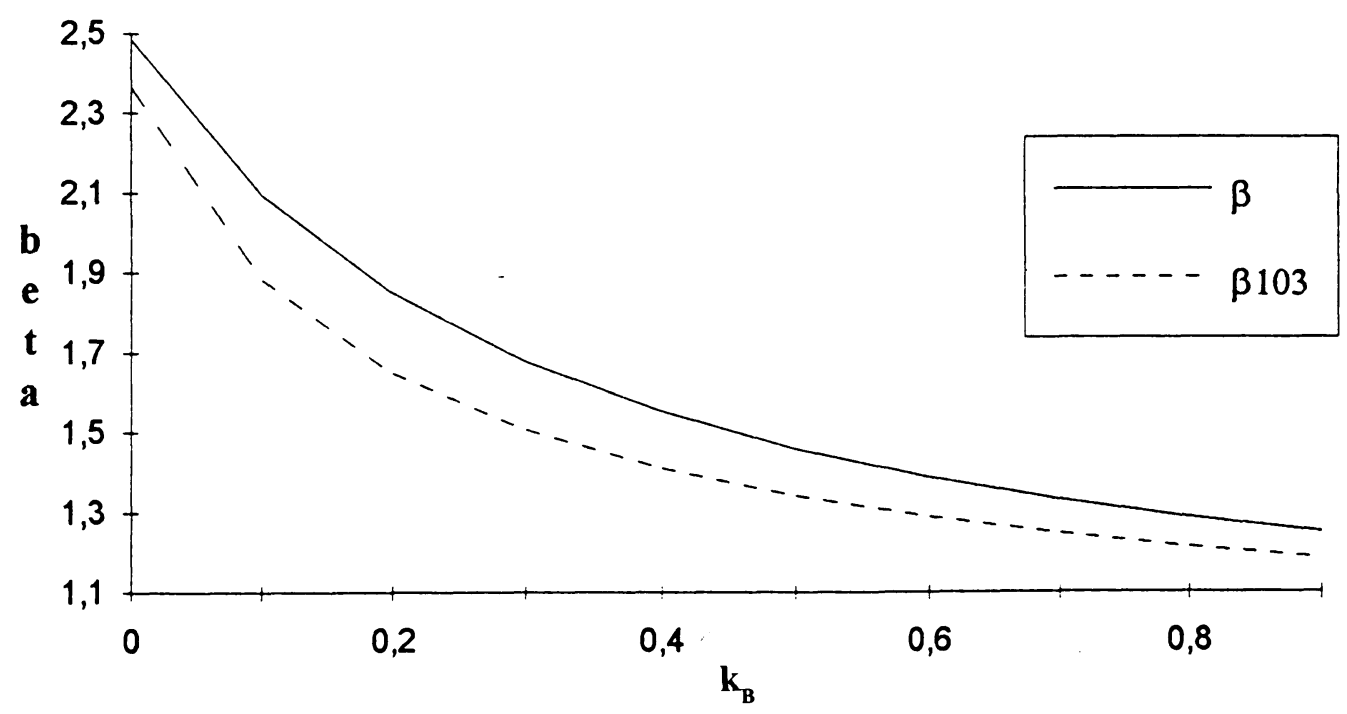

Gráfica II $\left(k_{a}=0,5\right)$

En la gráfica I hemos dado un valor fijo a $k_{A}\left(k_{A}=0\right)$ y hemos variado el valor de $k_{B}$. En las gráficas II y III, $k_{A}=0,5$ y $k_{A}=0,9$, respectivamente. Se puede comprobar que:

$$
k_{A}=1-R_{A} ; \quad k_{B}=1-R_{B}
$$

\section{CONCLUSIONES}

Los errores de la tabla 3.2, caso 1 , son sencillos de solventar, al ser el valor de los momentos de los pilares:

$$
\left(\frac{\mathrm{P}+\mathrm{P}_{1}}{2}\right) \frac{\delta}{2}
$$


TABLA III.

\begin{tabular}{|c|c|c|c|c|c|c|c|c|c|c|}
\hline$k_{A}$ & \multicolumn{10}{|c|}{0,9} \\
\hline$k_{B}$ & 0,0 & 0,1 & 0,2 & 0,3 & 0,4 & 0,5 & 0,6 & 0,7 & 0,8 & 0,9 \\
\hline$k l$ & 1,5283 & 1,7680 & 1,9822 & 2,1773 & 2,3532 & 2,5113 & 2,6519 & 2,7756 & 2,8234 & 2,9766 \\
\hline$\beta$ & 2,0555 & 1,7769 & 1,5843 & 1,4428 & 1,3349 & 1,2509 & 1,1846 & 1,1318 & 1,0895 & 1,0554 \\
\hline$\beta_{103}$ & 2,0439 & 1,6558 & 1,4572 & 1,3338 & 1,2489 & 1,1865 & 1,1385 & 1,1004 & 1,0693 & 1,0435 \\
\hline
\end{tabular}

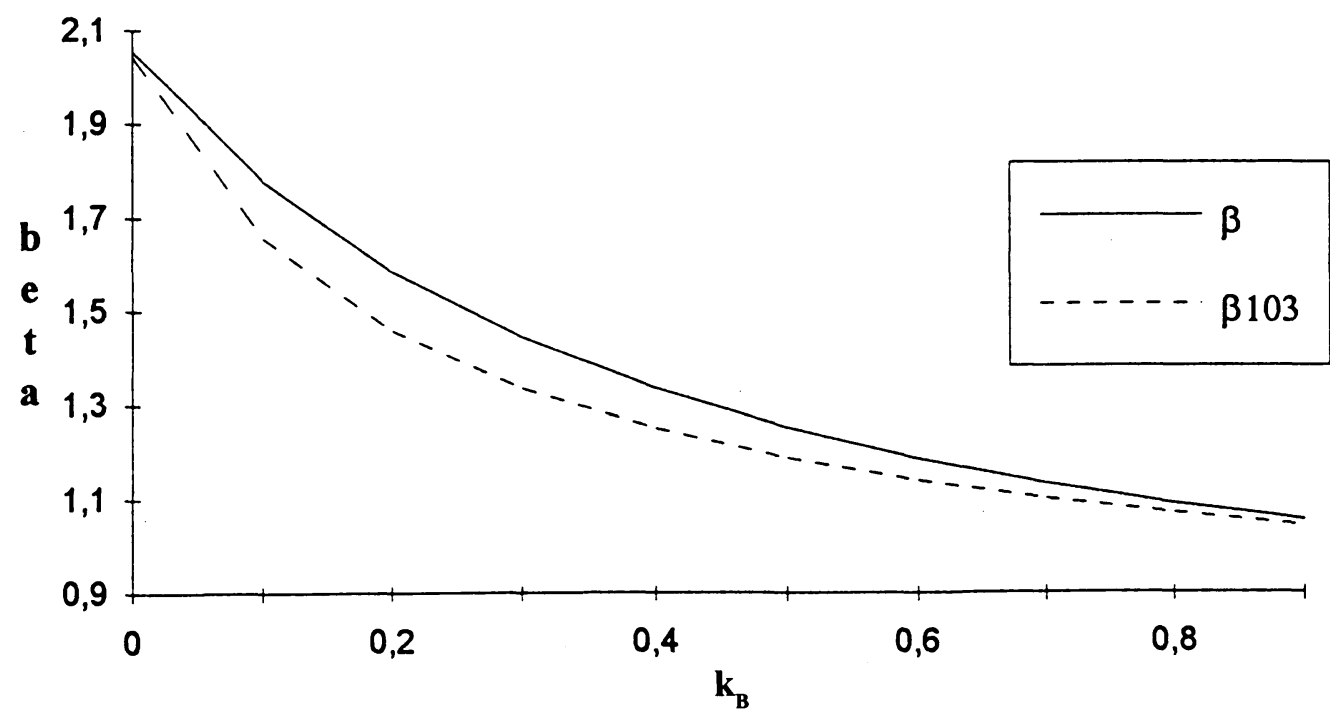

Gráfica III $\left(k_{a}=0,9\right)$

independiente de las longitudes de las barras, contando siempre con la simetría de medidas y perfiles.

El caso 2, contando con las mismas simetrías, no es independiente de las longitudes. Por lo que la solución de la corrección es bastante más complicada. Nosotros sólo hemos constatado el hecho.

En cuanto a las tablas 3.3 y 3.4 de la $M V-103$, los resultados nos parecen buenos dentro de los coeficientes de seguridad que se usan en el cálculo y la influencia del coeficiente de pandeo en el resultado final. 